\title{
A deficiência de tiamina e niacina como fator de risco para doenças neurológicas
}

\author{
The deficiency of thiamine and niacin as a \\ risk factor for neurological disease
}

Recebido em: 18/09/2018 Aceito em: $\quad 14 / 05 / 2019$
Nayrene Amorin Carvalho OLIVEIRA; Laryssa Alves MAGALHÃES; Maria Rosimar Teixeira MATOS; Gislei Frota ARAGÃO;

Tatiana Paschoalette Rodrigues BACHUR Universidade Estadual do Ceará. Av. Dr. Silas Munguba, 1700, Itaperi. CEP 60714-903. Fortaleza, Ceará, Brasil. E-mail:tatiana.bachur@uece.br

\section{ABSTRACT}

Niacin and thiamine, vitamins obtained through diet, are precursors of coenzymes of the intermediate metabolism. The objective of the present study was to review the literature on these vitamins in oxidative metabolism and its implications for neurological diseases. The methodology consisted of a bibliographic search in the Medline and Science Direct databases, using the descriptors "oxidative stress", deficiency, "basal metabolism", "nervous system", "thiamine" and "niacin". Ten articles were obtained for the production of the review and the studies showed that the deficiency of those vitamins can cause dysfunction in the central nervous system due to different alterations in oxidative metabolism.

Keywords: oxidative stress; thiamine; niacin; Central Nervous System

\section{RESUMO}

Niacina e tiamina, vitaminas obtidas por meio da dieta, são precursoras de coenzimas do metabolismo intermediário. O objetivo do presente trabalho foi realizar uma revisão da literatura a respeito dessas vitaminas no metabolismo oxidativo e suas implicações em doenças neurológicas. A metodologia consistiu na realização de uma busca bibliográfica nas bases de dados Medline e Science Direct, utilizando os descritores "oxidative stress", deficiency, "basal metabolism", "nervous system", "thiamine" e "niacin". Ao final, foram destacados dez artigos para a produção da revisão. Os estudos demonstraram que a deficiência das vitaminas niacina e tiamina pode causar disfunções no sistema nervoso central devido a falhas no metabolismo oxidativo.

Palavras-chave: estresse oxidativo; tiamina; niacina; Sistema Nervoso Central

\section{INTRODUÇÃO}

Tiamina e a niacina são vitaminas obtidas através da dieta, sendo hidrolisadas no estômago. Sua absorção ocorre no intestino delgado. A tiamina, após absorvida, pode ser transportada para os tecidos, onde atuará, em forma de piro- fosfato de tiamina (TPP), como coenzima para enzimas redox. A niacina pode ser encontrada nos alimentos na forma de nucleotídeos de nicotinamida (nicotinamida adenina dinucletídeo - NADH e fosfato de nicotinamida adenina dinucleotídeo - NADPH) e nicotinamida livre (NAm) (1-3). 
Estas vitaminas pertencentes ao complexo B atuam como coenzimas e favorecem o carreamento de elétrons passando, de forma reversível, por oxidação e redução em várias reações metabólicas. As reações de oxidação-redução envolvem a perda de elétrons por uma espécie química, que é oxidada, e o ganho de elétrons por outra espécie, que é reduzida $(1,4,5)$.

O estresse oxidativo, importante causa de dano celular, ocorre devido um desequilíbrio entre fatores pró-oxidantes e antioxidantes. O sistema de defesa antioxidante busca manter o processo oxidativo dentro dos limites adequados e passíveis de regulação, impedindo maiores danos. É importante relatar que o organismo está sujeito ao estresse oxidativo ocasionado por espécies reativas de oxigênio (ERO) provenientes do meio ambiente ou formadas no próprio organismo $(6,7)$.

$\mathrm{O}$ cérebro e o sistema nervoso central são mais propensos ao estresse e ao dano oxidativo, influenciando na ocorrência de doenças neurodegenerativas. $\mathrm{O}$ aumento do dano oxidativo, a disfunção mitocondrial e defeitos nas proteínas constituem condições que levam à morte celular de neurônios (2).

Diante da relevância da tiamina e da niacina no metabolismo, o objetivo do presente estudo consistiu na revisão da literatura a respeito da deficiência dessas vitaminas do complexo B no metabolismo oxidativo e suas implicações em doenças neurológicas.

\section{MÉTODO}

O presente trabalho consiste em uma revisão bibliográfica descritiva com abordagem qualitativa. Para atender ao objetivo do estudo, as produções científicas referentes ao tema foram pesquisadas nas bases de dados Science Direct e Medline usando os seguintes descritores: "oxidative stress", deficiency, "basal metabolism" e "nervous system" combinados com "niacin" e "thiamine". Os critérios de inclusão considerados foram: estudos publi- cados no período de 2011 a 2018, pesquisas in vivo e in vitro, publicações nos idiomas inglês e português, disponíveis na íntegra e cujo tema remetesse a doenças do sistema nervoso central e à deficiência das vitaminas estudadas. Foram excluídos artigos em duplicata, não disponíveis na íntegra, em idiomas diferentes dos considerados, bem como artigos que não abordavam a correlação da deficiência de niacina ou tiamina e doenças neurológicas. A partir da pesquisa, foram selecionados dez artigos, os quais foram utilizados para a produção dessa revisão bibliográfica.

\section{RESULTADOS E DISCUSSÃO}

Os principais resultados encontrados nos artigos analisados estão sumarizados no Quadro 1.

Níveis diminuídos de tiamina estão correlacionados com o processo de envelhecimento (8). Assim, deficiência de tiamina no cérebro causa uma cascata de eventos, incluindo comprometimento do metabolismo oxidativo, neuroinflamação e neurodegeneração, que são comumente observados em doenças neurodegenerativas, como doença de Alzheimer (DA), doença de Parkinson (DP) e doença de Huntington (DH) (9).

A doença de Alzheimer é uma doença neurodegenerativa progressiva e relacionada à idade, caracterizada pela deposição de placas senis beta-amiloides associadas a emaranhados neurofibrilares (10). Pacientes com doença de Alzheimer apresentam comprometimento da memória e declínio cognitivo devido à perda neuronal, principalmente no neocórtex e no hipocampo. Também é observada a grave redução do metabolismo da glicose no cérebro, pois há uma redução em três enzimas importantes dependentes de tiamina, transcetolase, complexo piruvato desidrogenase (PDHC) e complexo de $\alpha$-cetoglutarato desidrogenase (KGDHC), que funcionam nos principais passos do metabolismo da glicose, o qual ocorre predominantemente nas mitocôndrias (11). 
Quadro 1. Principais achados na literature, entre 2011 e 2018, sobre a deficiência de tiamina e niacina com consequências em doenças neurológicas.

\section{Autores, Ano}

T, et al., 2014

\begin{tabular}{l|l} 
& 5 \\
& 6 \\
& 7
\end{tabular}

Kim Y, Kim M, Park SH, Cho I,

Paik JK., 2018 dorff L, et al., 2016

Mouton-liger F, Rebillat AS, Gourmaud S, et al., 2015

1

3.
Ivanov AI, Malkov AE, Waseem 1.

krtchyan G, Graf A, Betten-

\section{Tipo de artigo/População}

5. Artigo original.

6. $\quad$ Estudo em seres humanos.

7. Indivíduos com acidente vascular cerebral (AVC) categorizados em grupo sem transtorno de ansiedade/depressão e grupo com transtorno de ansiedade/depressão.

9. Artigo original.

10. Estudo experimental.

11. Ratos Wistar fêmeas.

13. Artigo original.

14. Estudo experimental.

15. Camundongos C57BL/6 WT e PKR.

Artigo original.

Pan X, Fei G, Lu J, et al. 2016 Estudo em seres humanos. Pacientes com doença de Alzheimer (DA).

17. Artigo original.

Sang S, Pan X, Chen Z, et al. 18. Estudo experimental. 2018

19. Camundongos transgênicos APP/ PS1 e C57BL/6.

Sarkar S, Liachenko S, Paule $21 . \quad$ Artigo original. MG, et al, 2016.

22.

23.

Estudo experimental.

Ratos Sprague Dawley machos.
24. Artigo original.

25. Estudo experimental.

26. Camundongos Swiss machos.

2013

Sharma A, Bist R, Bubber P., 24.

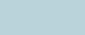

Zhang $Q$, Yang G, Li W, et al, 28 .

28. Artigo original.

2011.

29.

Estudo experimental. Camundongos $[57 \mathrm{BL} / 6 \mathrm{~J}$ machos adultos.

\begin{tabular}{l|ll}
$\begin{array}{l}\text { Zhang G, Ding H, Chen H, et } \\
\text { al., } 2013\end{array}$ & $\begin{array}{l}30 . \\
31 .\end{array}$ & $\begin{array}{l}\text { Artigo original. } \\
\text { Estudo em seres humanos. Pacien- } \\
\text { tes com sem depressão. }\end{array}$
\end{tabular}

\section{Principais resultados}

4. A deficiência de niacina pode causar disfunção mitocondrial com um acúmulo de lactato.

8. Indivíduos com AVC com transtorno de ansiedade/depressão demonstraram déficit de niacina.

12.

Níveis diminuídos de tiamina e/ou atividade da oxoglutarato desidrogenase prejudicada resultaram em envelhecimento e/ou ocorrência de doenças neurodegenerativas.

16. A deficiência de tiamina induziu estresse oxidativo e inflamação, bem como aumentou a ativação da proteína quinase $R$ (PKR) no tálamo e cerebelo.

0 metabolismo alterado de tiamina pode contribuir para a deficiência e neurodegeneração por perturbar 0 metabolismo da glicose no cérebro e induzir múltiplos fatores patogênicos na doença de Alzheimer, tornando a tiamina um potencial alvo para terapia da DA.

20. Camundongos em uma dieta deficiente de tiamina manifestaram declínio significativo de valores padrão de captação de imagens em múltiplas regiões cerebrais.

Observou que a deficiência de tiamina diminui gravemente o transportador de glicose (GLUT-1), que é expresso em células endoteliais, causando, assim, neurodegeneração das áreas cerebrais, ativando a micróglia que ultrapassa a barreira cerebral endotelial

27. A redução na atividade de enzimas antioxidantes, bem como o aumento de peroxidação lipídica e proteína carbonil após deficiência de tiamina, implicam em estresse oxidativo nas mitocôndrias cerebrais, podendo levar à neurodegeneração.

Observou que a administração de suplementação de tiamina reverte a acumulação das placas beta amiloides, sugerindo que a suplementação da tiamina na população idosa pode ser útil para prevenir ou retardar a formação de placas beta amiloides e beneficiar pacientes com doença de Alzheimer.

A tiamina livre e o monofosfato de tiamina foram significativamente menores em indivíduos com sintomas depressivos do que entre aqueles sem sintomas.
As mitocôndrias desempenham um papel fundamental no sistema nervoso central, pois são altamente dependentes de energia. Assim, a disfunção mitocondrial que ocorre devido a defeitos na reação de redução ou de fosforilação oxidativa, resulta em danos oxidativos no metabolismo e na diminuição da produção de energia que, consequentemente, leva a distúrbios neurológicos. Neurônios e astrócitos usam a glicólise e a fosforilação oxidativa para atender às suas demandas energéticas (12). Disfunções mitocondriais têm sido demonstradas como um evento inicial em doença de Alzheimer; 
assim, é possível que a disfunção mitocondrial contribua para a menor concentração de glicose cerebral e, portanto, hipometabolismo (4).

Pacientes com doença de Alzheimer exibem uma diminuição significativa no conteúdo de difosfato de tiamina, bem como 2-oxoglutarato dependente e desidrogenase dependente de difosfato de tiamina em diferentes áreas do cérebro, indicando fortes prejuízos no sistema de fosforilação da tiamina (13). A deficiência desta vitamina causa estresse oxidativo e perda neuronal específica regional em cérebros de animais e humanos $(12,14)$. Modelos animais e celulares, por meio da detecção de um amplo espectro de marcadores de estresse oxidativo, como heme oxigenase-1, superóxido dismutase, ferritina e malondialdeído aumentados no cérebro, mostraram que a deficiência de tiamina aumenta a produção de espécies reativas de oxigênio (ERO) e causa estresse oxidativo nas células cerebrais e neuronais. Além disso, foi observado que níveis aumentados de tiamina podem ter aplicação terapêutica em pacientes com doenças neurodegenerativas (14).

Experimentalmente, Sang e cols (2018) demonstraram que a redução do difosfato de tiamina, forma ativa da tiamina, prejudicou o metabolismo da glicose cerebral em camundongos, manifestando-se por meio de declínios significativos na captação de glicose em múltiplas regiões cerebrais de animais sujeitos a deficiência de tiamina na alimentação, em comparação com grupo controle (15).

Ivanov e cols (2014) observaram que a suplementação ou substituição de glicose no líquido cefalorraquidiano artificial por piruvato ou lactato modificou fortemente os parâmetros relacionados ao metabolismo energético acionado por atividade de rede dos neurônios. Sugeriram que durante a ativação da rede de neurônios, a glicólise estimula uma resposta significativa de NADPH e as mudanças no NAD citosólico e FAD mitocondrial (16).

A ingestão inadequada de proteínas e vitaminas favorece a deficiência de niacina e depleção de NAD. No entanto, a síntese de niacina e NAD a partir da via da quinurenina do metabolismo do triptofano pode compensar a ingestão alimentar prejudicada. Fármacos antidepressivos têm efeito anti-inflamatório, incluindo a redução de interferon-gama e, portanto, a inibição de indolamina 2,3-dioxigenase (IDO), a enzima limitante da velocidade da via da quinurenina. Portanto, pacientes com dieta pobre em micronutrientes, tratados com antidepressivos, estão em risco de desenvolver deficiência de niacina NAD com possível desenvolvimento de sintomas neuropsiquiátricos associados (17).

Buscando compreender a influência da deficiência de niacina no sistema nervoso central, Kim e cols (2018) analisaram a ingestão nutricional dessa vitamina em pacientes com acidente vascular cerebral (AVC) de acordo com seu grau de ansiedade/depressão. A ingestão de niacina foi significativamente maior nos pacientes com AVC sem ansiedade/depressão do que naqueles com AVC, acometidos por ansiedade/depressão. Portanto, o grupo de pacientes com AVC sem ansiedade/ depressão apresentou índice de qualidade de vida significativamente maior em comparação ao grupo que apresentou AVC com um problema de ansiedade/depressão (18).

Níveis diminuídos de tiamina estão associados ao processo de envelhecimento e neurodegeneração. A deficiência de tiamina pode causar estresse oxidativo, e autofagia no cérebro. A interação do estresse oxidativo, estresse de retículo endoplasmático e autofagia contribui para a neurodegeneração mediada por deficiência de tiamina (9).

Xie e cols (2013) demonstraram que o estresse oxidativo foi mais expressivo em neurites perto de placas beta-amiloides, propagadas para corpos celulares, e precedido de ativação de caspases, levando à morte celular dentro de $24 \mathrm{~h}$. A deficiência de niacina levou ao estresse oxidativo local em torno das placas e contribuiu para a toxicidade de longo alcance e morte neuronal seletiva na doença de Alzheimer (19)

De acordo com Ansari e cols (2010), o estresse oxidativo encontra-se mais localizado nas sinapses, com níveis que aumentam e evoluem juntamente com a doença de Alzheimer, implicando no envolvimento de estresse oxidativo na perda sináptica relacionada à doença de Alzheimer. Os níveis de antioxidantes cerebrais não são suficientes para neutralizar a oxidação, embora esses antioxidantes, 
como a niacina possam ser profiláticos no início do processo da doença de Alzheimer (20).

Pacientes com doença de Alzheimer têm hipometabolismo de glicose cerebral. Pan e cols (2016) observaram que os pacientes com doença de Alzheimer apresentam níveis baixos de difosfato de tiamina (TDP), sendo essa vitamina uma coenzima importante para o metabolismo da glicose. Avaliar os metabólitos da tiamina sanguínea pode ser importante para distinguir a doença de Alzheimer de outras doenças com sintomatologias semelhantes. Assim, o metabolismo alterado de tiamina pode contribuir para a deficiência e neurodegeneração por perturbar o metabolismo da glicose no cérebro e induzir múltiplos fatores patogênicos na doença de Alzheimer, tornando a tiamina um potencial alvo para terapia de doença de Alzheimer (21).

É sabido que a deficiência prolongada de vitamina B1 (tiamina) pode levar a distúrbios neurológicos como a Encefalopatia de Wernicke (EW) e Síndrome de Wernicke-Korsakoff (SWK) em humanos, pois a deficiência de tiamina causa extravasamento vascular, ruptura da barreira hematoencefálica e perda neuronal do diencéfalo e tronco cerebral (11). Sarkar e cols (2016) observaram que a deficiência de tiamina diminui gravemente o transportador de glicose (GLUT-1), que é expresso em células endoteliais, causando, assim, neurodegeneração das áreas cerebrais, ativando a micróglia que ultrapassa a barreira cerebral endotelial $(11,22)$.

Zhang e cols (2011) induziram a deficiência de tiamina em indivíduos com comprometimento leve de cognição e observaram que a administração de suplementação de tiamina reverteu a acumulação das placas beta amiloides, sugerindo que a suplementação da tiamina na população idosa pode ser útil para prevenir ou retardar a formação de placas beta-amiloides e beneficiar pacientes com doença de Alzheimer. Além disso, a modulação de enzimas dependentes de tiamina pode ser uma via potencial para o tratamento da doença de Alzheimer (23).

\section{CONCLUSÃO}

As vitaminas niacina e tiamina estão envolvidas nas principais vias de regulação das mitocôndrias, como o metabolismo de aminoácidos, ácidos graxos, e a reação de oxidação-redução, essenciais para o desenvolvimento celular normal. A deficiência de tiamina pode levar ao desenvolvimento de neuropatias, como doença de Alzheimer, doença de Parkinson e doença de Huntington. Níveis deficientes de niacina podem levar à inflamação no SNC, maior estresse oxidativo, resultando em disfunção mitocondrial, além de afetar negativamente a qualidade de vida de pacientes com AVC que possuem transtornos de ansiedade/depressão.

Assim, a deficiência dessas vitaminas pode configurar uma das causas para os distúrbios mitocondriais e, consequentemente, distúrbios do sistema nervoso central e em diversos processos metabólicos, comprometendo a homeostasia. Portanto, é fundamental que essas vitaminas estejam presentes na alimentação a fim de prevenir doenças metabólicas e neurológicas ou minimizar seus sintomas.

\section{REFERÊNCIAS}

1. Cozzolino SMF, Cominetti C. Bases bioquímicas e fisiológicas da nutrição: nas diferentes fases da vida, na saúde e na doença. Barueri: Manole. 2013.

2. Cozzolino SMF. Biodisponibilidade de Nutrientes. 4 ed. Barueri: Manole. 2012.

3. Vannucchi H, Cunha SFC. Funções plenamente reconhecidas de nutrientes vitaminas do complexo B: tiamina, riboflavina, niacina, piridoxina, biotina e ácido pantotênico [on line]. Ilsi Brasil; 2009. Disponível em: http://ilsi. org/brasil/wp-content/uploads/sites/9/2016/05/09-Complexo-B.pdf .
4. Gemelli T, Andrade RB, Castro AL, Garcia L, Funchal C. Estresse oxidativo como fator importante na fisiopatologia da doença de alzheimer. Rev Uniarara. 2013;16(1):6778. DOI: $10.25061 / 2527-2675 / \operatorname{ReBraM} / 2013 . v 16 i 1.43$.

5. Nelson DL, Cox MM. Princípios de bioquímica de Lehninger. Porto Alegre: Artmed, 2011. 6. ed. Porto Alegre: Artmed, 2014.

6. Barbosa KBF, Costa NMB, Alfena SRCG, Paula SO, Minim VPR, Bressan J. Estresse oxidativo: conceito, implicações e fatores modulatórios. Rev. Nutr. 2010;23(4):629643. DOI: $10.1590 / \mathrm{S} 1415-52732010000400013$ 
7. Barreiros ALBS, David JM, David JP. Estresse oxidativo: relação entre geração de espécies reativas e defesa do organismo. Quím. Nova. 2006;29(1):113-123. DOI: $10.1590 / \mathrm{S} 0100-40422006000100021$.

8. Zhang G, Ding H, Chen H, Ye X, Li H, Lin X, Ke Z. Thiamine nutritional status and depressive symptoms are inversely associated among older Chinese adults. J Nutr. 2013;143(1):53-58. DOI: 10.3945/jn.112.167007.

9. Liu D, Ke Z, Luo J. Thiamine Deficiency and Neurodegeneration: the Interplay Among Oxidative Stress, Endoplasmic Reticulum Stress, and Autophagy. Mol Neurobiol 2017; 54(7):5440-5448. DOI: 10.1007/ s12035-016-0079-9.

10. Hohsfield LA, Humpel C. Intravenous infusion of monocytes isolated from 2-week-old mice enhances clearance of Beta amyloid plaques in an Alzheimer mouse model. PLoS One. 2015;10(4):1-18. DOI: 10.1371/journal. pone. 0121930 .

11. Gross AL, Manly JJ, PA J, Johnson JK, Park LQ, Mitchell MB, Melrose RJ, Inouye SK, Mclaren DG. Cortical signatures of cognition and their relationship to Alzheimer's disease. Brain Imaging Behav. 2013;6(4):584-598. DOI: $10.1007 / \mathrm{s} 11682-012-9180-5$.

12. Sharma A, Bist R, Bubber P. Thiamine deficiency induces oxidative stress in brain mitochondria of Mus musculus. J Physiol Biochem. 2013;69(3):539-546. DOI: 10.1007/ s13105-013-0242-y.

13. Mkrtchyan G, Graf A, Bettendorff L, Bunik V. Cellular thiamine status is coupled to function of mitochondrial 2-oxoglutarate dehydrogenase. Neurochem Int. 2016;101:66-75. DOI: 10.1016/j.neuint.2016.10.009.

14. Mouton-Liger F, Rebillat AS, Gourmaud S, Paquet C, Leguen A, Dumurgier J, Bernadelli P, Taupin V, Pradier L, Rooney T, Hugon J. PKR downregulation prevents neurodegeneration and beta-amyloid production in a thiamine deficient model. Cell Death Dis. 2015;6(1):e1594. DOI: $10.1038 /$ cddis.2014.552.

15. Sang S, Pan X, Chen Z, Zeng F, Pan S, Liu H, Jin L, Fei G, Wang C, Ren S, Jia F, Bao W, Zhou W, Guan Y, Zhang Y, Shi H, Wang Y, Yu X, Wang Y; Zhong C. Thiamine diphosphate reduction strongly correlates with brain glucose hypometabolism in Alzheimer's disease, where as amyloid deposition does not. Alzheimers Res Ther. 2018;10(1):1-13. DOI: 10.1186/s13195-018-0354-2.

16. Ivanov AI, Malkov AE, Waseem T, Mukhtarov M, Buldakova S, Gubkina O, Zilberter M, Zilbe Y. Glycolysis and oxidative phosphorylation in neurons and astrocytes during network activity in hippocampal slices. J Cereb Blood Flow Metab. 2014;(34)397-407. DOI: 10.1038/ jcbfm.2013.222.

17. Viljoen M, Swanepoel A, Bipath P. Antidepressants may lead to a decrease in niacin and NAD in patients with poor dietary intake. Med Hypotheses. 2015;84(3):178182. DOI: 10.1016/j.mehy.2014.12.017.

18. Kim Y, Kim M, Park SH, Cho I, Paik JK, Association of the Anxiety/Depression with Nutrition Intake in Stroke Patients. Clin Nutr Res. 2018;(7):11-20. DOI: 10.7762/ cnr.2018.7.1.11.

19. Xie H, Hou S, Jiangb J, Sekutowicz M, Kelly J, Bacskai BJ. Rapid cell death is preceded by amyloid plaque-mediated oxidative stress. Proc Natl Acad Sci USA. 2013;110(19):7904-7909. DOI: 10.1073/ pnas. 1217938110.

20. Ansari MA, Scheff SW. Oxidative stress in the progression of Alzheimer disease in the frontal córtex. Neuropathol Exp Neurol. 2010;69(2):155-167. DOI: 10.1097/ NEN.0b013e3181cb5af4.

21. Pan X, Fei G, Lu J, Jin L, Pan S, Chen Z, Wang C, Sang S, Liu H, Hu W, Zhang H, Wang Z, Tan Q, Qin Y, Zhang Q, Xie X, Ji Y, Cui D, Gu X, Xu J, Yu Y, Zhong C. Measurement of Blood Thiamine Metabolites for Alzheimer's Disease Diagnosis. EBioMedicine. 2016;3(1):155162 DOI: 10.1016/j.ebiom.2015.11.039.

22. Sarkar S, Lianchenko S, Paule MG, Bowyer J, Hanig JP. Brain endothelial dysfunction following pyrithiamine induced thiamine deficiency in the rat. NeuroToxicology. 2016;57(2):298-309 DOI: 10.1016/j.neuro.2016.10.014.

23. Zhang Q, Yang G, Li W, Fan Z, Sun A, Luo J, Ke Z. Thiamine deficiency increases -secretase activity and accumulation of -amyloid peptides. Neurobiology of Aging. 2011;32(1):42-53 DOI: 10.1016/j.neurobiolaging.2009.01.005. 